\title{
The Size of Events
}

\author{
Samuel Bayer \\ The MITRE Corporation
}

\section{Introduction}

The body of work in sentence semantics inspired by Davidson (Davidson, 1967) can be characterized by its diversity. On at least one matter, all the approaches agree: at some level of analysis, sentences denote sets of Davidsonian elements which correspond to states or occurrences in the world. I will call these elements eventualities for convenience. However, in many other ways this work (for instance, Kratzer (1989), Portner (1992), Lasersohn (1995), Parsons (1990), Schein (1993), Krifka (1992), Bayer (1995)) varies tremendously. It embraces work on both situations and events, and accounts based on thematic roles as well as accounts which rely on the Montegovian argument-order-based participant indexing strategy. These accounts vary on whether situations (partial worlds) are sufficient, or events (as ontological primitives) are required; on whether thematic roles are needed or desired in semantic composition; and on the properties of the sentence denotations themselves.

In this paper, I will examine one of these points of contention. A number of researchers (especially Kratzer (1989), Lasersohn (1995), but also Krifka (1989) and Portner (1992) to some extent) have proposed that the sets of eventualities which qualify as sentence denotations are persistent, in the sense given in (1):

(1) Given a universe of eventualities $D_{E}$, a set of eventualities $P \subseteq D_{E}$ is persistent if and only if for all $e, e^{\prime} \in D_{E}$ the following holds: whenever $e \leq e^{\prime}$ and $e \in P$, then $e^{\prime} \in P$. (Adapted from Kratzer (1989))

So if a persistent eventuality set contains an eventuality $e$, it also contains all eventualities "larger than $e$ ". This notion of persistence is inspired by notions of monotonic information growth in information states, as in the work of Kripke (1965) and its intellectual children such as Landman (1986).

In this paper, I will argue that persistence is not a requirement on sentence denotations. I will begin by making precise the $\leq$ relation and laying out my assumptions. Next, I will present a potential implementation of persistence, to make clear how proposals in the literature make the notion of persistence coherent. Then, I will review and critique previously presented motivations for persistence, and finally, I will present my own arguments against the constraint. 


\section{What does $\leq$ mean?}

I will assume a lattice-theoretic account of plurals, similar to Link (1983), Landman (1989), Lasersohn (1995), Ojeda (1991), Krifka (1992), etc. My generic version of these proposals involves at least:

- an associative, reflexive, commutative, idempotent join operator, which I will notate as +;

- a part-of relation $\leq$, defined so that for all $x$ and $y, x \leq y$ iff $x+y=y$;

- and the domain of individuals $D$, which is closed under the join operator.

There is a wide assortment of details involving the structure of this domain which are irrelevant to the present discussion. However, there are two matters which figure prominently. The first involves the scope of the lattice structures in question. Unlike Krifka (1989), for instance, I will not assume that these lattices are partitioned by ontological sort (object vs. eventuality, for instance), for reasons which will become clear in a moment. So while Krifka would have two parallel lattices, one for the domain of objects and one for the domain of eventualities, for the purposes of this discussion, there is one lattice, and $D_{E} \subseteq D$.

The second issue which I must take a stand on is the nature of atomhood. Roughly, an atom is a smallest element, but accounts differ on what set an atom is a smallest element of. Accounts of atomhood can be either what I'll call ontologically general or ontologically specific. I present the ontologically general notion of atomhood first:

(2) There is a subset of $D$ of atomic individuals $A t$, defined so that for all $x$ in At, $\neg \exists y(y \in D \wedge y \leq x \wedge y \neq x)$ (there are no elements in $D$ smaller than $x)$

Under this definition, atoms are smallest elements of the entire domain of individuals. This definition has a very important consequence: no atom of one class can be the sum of (among other things) atoms of another class. So limbs are not $\leq$ bodies, and following Barker (1992), committee members are not $\leq$ committees. That is, member-of and physical-part-of are not instantiations of $\leq$, and $A t$ is a set of individuals. In the ontologically specific version, in contrast, $A t$ is a relation between properties and individuals:

(3) For any property $P$, there is a subset of $D$ of atomic individuals $A t(P)$, defined so that for all $x$ in $A t(P), \neg \exists y(y \in A t(P) \wedge y \leq x \wedge y \neq x)$ (there are no elements in $A t(P)$ smaller than $x$ )

This definition is much more limited in its scope; in particular, it partitions atomhood according to fine-grained ontological sort. So committee members might be $\leq$ committees (since there can be no committee atom which has another smaller 
committee individual as a part, but no restrictions are imposed on the relationship between committee atoms and elements of another sort), limbs might be $\leq$ bodies, raising a fork can be $\leq$ eating a meal (this is fundamentally a part of the lumping constraint of Kratzer (1989)), and participants can be $\leq$ events (cf. Kratzer (1989) again). Because this account is more restricted with respect to atomhood, it is accordingly more general with respect to information growth. If an atomic eventuality of raising a fork can be part of an atomic eventuality of eating a meal, then this notion of "is a larger situation than" can double as the notion of "is more informative than" (that is, makes a superset of claims about the state of the world). Persistence thus relies on this ontologically specific notion of atomhood, since it is intended to reflect concerns of information growth. Many accounts of eventuality semantics adopt this ontologically specific definition of atomhood; so in this paper, $\leq$ for persistence relies on the ontologically specific lattice-theoretic notion of atomhood.

Finally, I will make two crucial assumptions in the remainder of this paper. First, I will adopt the uncontroversial assumption that a sentence is true iff its denotation is a nonempty set of eventualities:

\section{Assumption 1}

A sentence $S, \llbracket S \rrbracket \subseteq D_{E}$, is true iff $\llbracket S \rrbracket \neq \emptyset$.

Second, I will assume a set of what I'll call basic Davidsonian relations $\mathcal{D} \mathcal{R}_{\text {basic }}$ which yield sets of (ontologically specific) atoms:

\section{Assumption 2}

There is a set of relations $\mathcal{D} \mathcal{R}_{\text {basic }}$ such that for any relation $R^{n} \in \mathcal{D} \mathcal{R}_{\text {basic }}$ and for any $x_{1}, \ldots, x_{n-1}$, if $P=\left\{e \mid R^{n}\left(x_{1}\right) \ldots\left(x_{n-1}\right)(e)\right\}$, then $A t(P)=$ $P$ (that is, $P$ is a set of ontologically specific atoms).

So once we saturate all but the final eventuality argument of any element of $\mathcal{D} \mathcal{R}_{\text {basic }}$, the resulting set of eventualities contains no elements which are an (ontologically specific) lattice-theoretic part of another element in the same set. In other words, these relations map individuals into sets of minimal eventualities of the given relation; so if touch' is one of these relations, touch' $\left(\right.$ michael $\left.^{\prime}\right)($ latoya') is that set of eventualities in which LaToya touches Michael and nothing else happens. Notice that I do not identify $\mathcal{R}_{\text {basic }}$ with the set of lexical Davidsonian denotations; I will address the relationship between these two sets in the next section.

\section{How can persistence be implemented?}

Before we examine the validity of the persistence constraint, I want to examine how persistence might be implemented, since there's no sense in considering persistence unless it's implementable. As an illustration of the implementation of persistence, let's consider two accounts of atomic event descriptions, one persistent and the other not. In the persistent account, the denotation of the verb is based, in part, on an element of $\mathcal{D} \mathcal{R}_{\text {basic }}$ : 
(6) 【embrace $\rrbracket=\lambda y \lambda x \lambda e\left[\exists e^{\prime}\left(e^{\prime} \leq e \wedge e m b r a c e^{\prime}(j)(l)\left(e^{\prime}\right)\right)\right]$, where embrace $^{\prime} \in$ $\mathcal{D} \mathcal{R}_{\text {basic }}$

$$
\llbracket \text { LaToya embraced Jermaine } \rrbracket=\lambda e\left[\exists e^{\prime}\left(e^{\prime} \leq e \wedge \operatorname{embrace}(j)(l)\left(e^{\prime}\right)\right)\right]
$$

This account obeys the persistence constraint in (1) above. If some eventuality $e$ is an element of the sentence denotation in (7), it is there because some part of it is a minimal eventuality $e^{\prime}$ of Latoya embracing Jermaine. Since the $\leq$ relation is transitive, any eventuality of which $e$ is a part will also have $e^{\prime}$ as a part, so it will also be in the denotation in (7).

On the other hand, on a non-persistent (in fact, minimal) account, the denotation of the verb is drawn directly from $\mathcal{D} \mathcal{R}_{\text {basic }}$ :

$$
\llbracket \text { embrace } \rrbracket=e m b r a c e^{\prime}, \text { where } e m b r a c e^{\prime} \in \mathcal{D} \mathcal{R}_{\text {basic }}
$$

$$
\llbracket \text { LaToya embraced Jermaine } \rrbracket=\lambda e\left[\operatorname{embrace}^{\prime}(j)(l)(e)\right]
$$

This account is non-persistent because Assumption 2, which governs the properties of elements of $\mathcal{D} \mathcal{R}_{\text {basic }}$, is in direct conflict with (1); the former rules out elements which have other elements in the same set as proper parts, while the latter requires them.

\subsection{A potentially persistent account of quantified sentences}

With the persistent atomic account in hand, I turn now to possible persistent accounts of quantified sentences. We begin with monotone increasing determiners like at least three:

(10) 【At least three musicians embraced Jermaine $\rrbracket=$

$$
\lambda e\left[\geq 3 x\left(\text { musician }^{\prime}(x) \wedge x \leq e \wedge \exists e^{\prime}\left(e^{\prime} \leq e \wedge \operatorname{embrace}^{\prime}(j)(x)\left(e^{\prime}\right)\right)\right)\right]
$$

This denotation is persistent. If some eventuality contains at least three musicians embracing Jermaine as parts, every larger eventuality will contain at least three musicians embracing Jermaine as well. We move on to universal quantification. Kratzer proposes the following as an initial account:

(adapted from Kratzer (1989))

【Every musician embraced Jermaine】 =

$$
\lambda e\left[\forall x\left(\left(\operatorname{musician}^{\prime}(x) \wedge x \leq e\right) \rightarrow \exists e^{\prime}\left(e^{\prime} \leq e \wedge \operatorname{embrace}(j)(x)\left(e^{\prime}\right)\right)\right)\right]
$$

This denotation is not persistent. For instance, if $e$ is the eventuality which encompasses just the events on the stage of the Orpheum on Tuesday night, and every musician in that situation embraced Jermaine, then $e$ will be in this denotation, but some larger eventuality $e^{\prime}$ which includes other musicians who do not embrace Jermaine will not be in this denotation. An analogous account of monotone decreasing determiners fails for a similar reason: 
(12) (inspired by Lasersohn (1995))

【Fewer than three musicians embraced Jermaine》 = $\lambda e\left[<3 x\left(\right.\right.$ musician $\left.\left.^{\prime}(x) \wedge x \leq e \wedge \exists e^{\prime}\left(e^{\prime} \leq e \wedge \operatorname{embrace}^{\prime}(j)(x)\left(e^{\prime}\right)\right)\right)\right]$

If $e$ is an eventuality which contains no more than three musicians embracing Jermaine (if, for instance, there are no such subeventualities), $e$ will be in this denotation, but larger eventualities $e^{\prime}$ which contain more musicians and more embracings may not. So if we desire persistence, we require a different account.

\subsection{A better quantified account}

In both cases here, the condition $x \leq e$ is the source of the trouble. The problem appears to be that if the individuals being quantified over are ontologically specific parts of the eventualities being referenced, larger eventualities will have more individuals, and thus run the risk of exceeding the upper bound imposed by determiners like fewer than three. The obvious answer is to remove this dependency. The justification for this move is concisely motivated by Lasersohn (1995), who points out that CNs in utterances commonly do not range over the entire denotation of the CN itself; that is, Every woman ate is commonly interpreted to quantify over a set of women whose relevance is previously determined, not every woman in the world under consideration. In other words, the appropriate contextual restriction on quantification is not necessarily a function of the denotation of the current utterance.

I do not intend to propose or adopt a theory of context here, only to exploit this observation in a revised account of persistence in quantification. I will assume that whatever theory of context we choose makes available to us a set of elements $\mathcal{R}$ consisting of individuals determined to be relevant in the current discourse. The elements in $\mathcal{R}$ might be individuals previously mentioned in discourse, or individuals in the discourse participants' common ground. The intersection of $\mathcal{R}$ with the set of musicians, for instance, will determine the independently relevant musicians; similarly for all other sets of individuals. This proposal is similar to ones made by Kratzer (1989) and Lasersohn (1995). ${ }^{1}$

Now consider how this proposal plays out in a revised account of persistent quantification. We begin with the case of every:

$$
\begin{aligned}
& \llbracket \text { Every musician embraced Jermaine } \rrbracket= \\
& \lambda e\left[\forall x\left(\left(\operatorname{musician}^{\prime}(x) \wedge x \in \mathcal{R}\right) \rightarrow \exists e^{\prime}\left(e^{\prime} \leq e \wedge \operatorname{embrace}(j)(x)\left(e^{\prime}\right)\right)\right)\right]
\end{aligned}
$$

This denotation, in contrast to the previous attempt; is persistent. For a given set of contextually relevant musicians, each eventuality in this denotation contains an eventuality of embracing Jermaine for each musician, and any larger eventuality will contain at least these eventualities as well. In other words, since we hold the set of contextually relevant musicians fixed and independent of the size of the eventuality, persistence is assured. Consider now the case of fewer than three: 


$$
\begin{aligned}
& \llbracket \text { Fewer than three musicians embraced Jermaine } \rrbracket= \\
& \lambda e\left[<3 x\left(\operatorname{musician}^{\prime}(x) \wedge x \in \mathcal{R} \wedge \exists e^{\prime}\left(e^{\prime} \leq e \wedge \text { embrace }(j)(x)\left(e^{\prime}\right)\right)\right)\right]
\end{aligned}
$$

Unlike the case of every, this denotation is not yet persistent. The problem is that we've fixed the set of musicians but not the set of embracing eventualities. So for instance, an eventuality which contains no embracings at all is an element of this denotation, but clearly larger eventualities might contain more than three embracings of Jermaine by relevant musicians. In this case, we must assume that $\mathcal{R}$ includes all contextually relevant eventualities as well, in this case all contextually relevant eventualities of relevant musicians embracing Jermaine:

$$
\begin{aligned}
& \llbracket \text { Fewer than three musicians embraced Jermaine } \rrbracket= \\
& \lambda e\left[<3 x\left(\operatorname{musician}^{\prime}(x) \wedge x \in \mathcal{R} \wedge\right.\right. \\
& \quad \forall e^{\prime}\left(\left(e^{\prime} \in \mathcal{R} \wedge \exists y\left(\operatorname{embrace}^{\prime}(y)(x)\left(e^{\prime}\right)\right)\right) \rightarrow e^{\prime} \leq e\right) \wedge \\
& \left.\left.\quad \exists e^{\prime}\left(e^{\prime} \leq e \wedge \operatorname{embrace}^{\prime}(j)(x)\left(e^{\prime}\right)\right)\right)\right]
\end{aligned}
$$

This denotation is now persistent. By assuring that all relevant embracings are included, we guarantee that any larger eventuality cannot "add" eventualities which might falsify the utterance.

\section{Why persistence (and why not)?}

As I've illustrated, one way to create a persistent account of certain quantified expressions is to make certain minimal assumptions about the nature of context. Kratzer (1989) comments that "once we recognize the importance of quantifier restrictions contributed by the utterance situation, we are not forced anymore to assume that propositions expressed by sentences involving universal quantifiers or definite descriptions are not persistent (p. 623)." Kratzer subsequently argues that the persistence constraint has implications for the semantics of counterfactuals. In particular, the best way to guarantee the persistence of counterfactuals is to require that the denotations of counterfactuals contain only maximal situations (that is, worlds), and that this latter property has useful implications for the account of counterfactuals.

But Kratzer really presents no arguments for persistence here. Let's consider counterfactuals first. If the same property of the denotations of counterfactuals (namely, containing only maximal situations) both has useful consequences for the semantics of counterfactuals and guarantees persistence, this fact doesn't count as an argument for persistence; the property in question is needed independently, and the fact that persistence arises as a side effect is irrelevant. True, adopting persistence as a general condition has the property of inducing an account which entails the appropriate semantics for counterfactuals, but but adopting maximal situations specifically for counterfactuals does so more directly.

Second, if persistence holds (and we've shown that it can), then the denotation $P$ of each sentence can be generated from the set of smallest elements of $P$ 
(that is, $A t(P)$ ). If this is the case, it's certainly more perspicuous to use $A t(P)$ instead of $P$ as the sentence denotation. Kratzer's first comment documents the opposition to this idea; for her, persistence is something which we ought to adopt unless forced otherwise. The most clearly stated motivation I am aware of for this conviction is found in Lasersohn (1995):

[In choosing between a non-persistent and a persistent account of the semantics of together,] I prefer the second approach, out of a conviction that the truth of all sentences must be persistent. This idea has its roots in the same intuition which gives rise to the view that logic is monotonic; conclusions which follow logically from limited information continue to follow as more complete information is gained. (p. 227)

So Lasersohn draws an analogy between information states and sentence denotations; Kratzer makes the same argument. The idea is that if information states are persistent, then sentence denotations ought to be as well.

However, I don't think this analogy works, and the reason has to do with the way information states and sentence denotations are related. Information states are borne by participants in discourse, and utterances transmitted among these participants affect their internal information states. Lasersohn's analogy would assign the same properties to the internal states of discourse participants and to the messages that these participants exchange; however, there is ample precedent that in the sort of situation we're considering here, this redundancy is not needed.

For instance, consider the Gricean conversational maxims and their relevance to the following well-known exchange:
A: How good a student is Smythe?
B: Well, she has excellent penmanship.

Following Grice, speaker $A$ is entitled to infer that Smythe is not a very good student (or, at the very least, that speaker B believes this), because speaker B is constrained by conversational principles to answer the question directly and affirmatively if possible. The conversational principles which guide this inference are part of the "rules of the game" which both speakers share, but they are in no way part of the denotation of speaker B's utterance. I propose that persistence is just another "rule of the game". So when a speaker utters a statement like Every musician embraced Jermaine, that speaker is assumed to be conforming to the requirement that there is some contextually refined interpretation of this utterance which the hearer can incorporate into her information state without fear of subsequent falsification. In other words, one requirement for cooperative interaction is that speakers attempt to ensure that the hearers can preserve the persistence of their information states. In such an account, there's no need to assume persistence of the sentence denotations themselves, since persistence is a property of the information states of the relevant discourse participants. 


\section{Against persistence in sentence denotations}

So far, I've illustrated how persistence might be possible, but rejected the motivations which have appeared in the literature. In this section, I will argue that persistence is not simply unmotivated, but that it also leads to unwelcome consequences for semantic composition. These unwelcome consequences arise both with respect to the internal composition of sentence denotations and external sentential reference. This argument crucially relies on the appropriate analysis of intersective modification, which is where I begin my discussion.

\subsection{Intersective modification}

The analysis of intersective modification is actually one of the strongest arguments in favor of Davidsonian semantics. This argument originates in Davidson (1967), and is elaborated on in Parsons (1990), Landman (1993), and Bayer (1995). In fact, the term intersective modification is something of a giveaway, because it foreshadows the appropriate analysis of a subclass of modifiers which are typically non-scalar, non-scopal and extensional. In particular, the order of these modifiers can be permuted (within the bounds of syntactic permissiveness) without affecting truth conditions; dropping a modifier yields a larger denotation set; and any two sequences of drops which result in the same set of remaining modifiers also yields the same denotation. I illustrate with nominal modifiers:

【forty-year-old blue-eyed American man $=$ 【forty-year-old American blue-eyed man $\rrbracket=$ 『blue-eyed forty-year-old American man】 etc.
a. 【forty-year-old blue-eyed American man $\subseteq$ $\llbracket$ forty-year-old American man $\subseteq \subseteq$ $\llbracket$ American man $\subseteq$ 【man】
b. 【forty-year-old blue-eyed American man $\subseteq$ $\llbracket$ forty-year-old blue-eyed man $\subseteq \subseteq$ $\llbracket$ forty-year-old man $\subseteq$

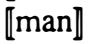

etc.

Landman (1993) argues at length that these properties of permutation and drop follow only from an analysis in which these modifiers are intersected with each other and with the modifiee. ${ }^{2}$ Crucial to this analysis is the requirement that the head and modifier bear the same semantic type at some level, since this is the only way they can be intersected: 
(19)

$$
\begin{aligned}
& \text { 【forty-year-old blue-eyed American man } \rrbracket= \\
& \llbracket \text { forty-year-old } \cap \cap \llbracket \text { blue-eyed } \rrbracket \cap \llbracket \text { American } \rrbracket \cap \llbracket \operatorname{man} \rrbracket= \\
& \lambda x\left[\text { forty-year-old }(x) \wedge \text { blue-eyed }(x) \wedge \operatorname{american}^{\prime}(x) \wedge \operatorname{man}^{\prime}(x) \rrbracket\right.
\end{aligned}
$$

If the only adequate analysis of the permutation and drop properties of this subclass of modifiers is given in terms of intersection, and if these intersective modifiers must be sets of the same sorts of elements that their modifiees are sets of, then if we can find verbal modifiers with the same set of properties, then we should conclude that the same analysis applies. And if we can show that none of the surface verbal arguments (that is, the eventuality participants) are the appropriate sort of thing to intersect with the modifiees, then we must conclude that there is a "hidden" (Davidsonian) argument which characterizes these individuals. Of course, a subclass of verbal modifiers exhibit just the desired behavior. In (20) we illustrate permutation:

(20) 『Jermaine prayed at the altar for ten minutes

in honor of Janet's wedding $\rrbracket=$

『Jermaine prayed at the altar in honor of

Janet's wedding for ten minutes》=

『Jermaine prayed for ten minutes at the altar

in honor of Janet's wedding $\rrbracket=$

【In honor of Janet's wedding, Jermaine prayed

at the altar for ten minutes》

etc.

In (21) we illustrate drop. In order not to prejudice the result, we reinterpret the subset/superset relationship as implication:

a. 【Jermaine prayed at the altar for ten minutes

in honor of Janet's wedding $\rrbracket \rightarrow$

『Jermaine prayed at the altar for ten minutes》 $\rightarrow$

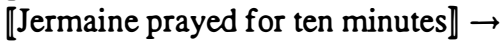

【Jermaine prayed》

b. 『Jermaine prayed at the altar for ten minutes in honor of Janet's wedding $\rightarrow$

『Jermaine prayed for ten minutes in honor of Janet's wedding》 $\rightarrow$

『Jermaine prayed in honor of Janet's wedding》 $\rightarrow$

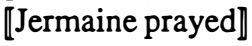

etc.

Finally, I will support the claim that none of the eventuality participants are appropriate candidates for intersection. Consider the modifier in honor of Janet's wedding. The sole "core" participant in the praying event in question is Jermaine, but Jermaine is not the sort of individual which is in the set of things which are in 
honor of Janet's wedding, as illustrated in (22). Here we see that nominal cases of intersective modification support predication between an element of the head and any one of the modifiers, but the verbal case supports predication only between the nominalization praying and the PP, but not between Jermaine and the PP: ${ }^{3}$
a. That man is forty years old/blue-eyed/American.
b. *Jermaine was in honor of Janet's wedding.
c. The praying was in honor of Janet's wedding.

I conclude, therefore, that verbal modifiers are sets of events, and that verbs have a "hidden" Davidsonian argument position which these modifier denotations are intersected with.

\subsection{The central counterargument}

At this point, I turn to the central counterargument. Essentially, I will argue that persistent denotations compromise intersective verbal behavior because the sets which are intersected are "too large". In this argument, I will contrast persistent denotation sets $P$ with their generating sets $A t(P)$. In order to cast the persistent alternative in the best possible light, I will restrict the scope of persistence, on the assumption that the smaller these persistent event sets are, the less their size can compromise intersective behavior. The particular restriction I will assume is that persistence is enforced only with respect to spatiotemporal locations. That is, I revise (1) as follows:

(23) Given a universe of eventualities $D_{E}$, a set of eventualities $P \subseteq D_{E}$ is persistent if and only if for all $e, e^{\prime} \in D_{E}$ the following holds: whenever $e \leq e^{\prime}$ and the spatiotemporal location of $e=$ the spatiotemporal location of $e^{\prime}$ and $e \in P$, then $e^{\prime} \in P$.

This revision is reminiscent of a number of restrictions imposed on persistence in the literature; cf. for instance, Krifka (1989).

With this background, I turn to our scenario. Jermaine prays as in (20) and (21) above, but he simultaneously pictures himself in a tropical paradise due to the long winter. I will assume, crucially, that these two minimal events are distinguishable and occur at the same spatiotemporal location. Call the maximal event at that location $e_{\max }$. We have the following denotations:

a. $\llbracket$ Jermaine prayed $\rrbracket=$ $\left\{e_{\text {pray }}, e_{\text {pray }}+e_{\text {fantasy }}, \ldots, e_{\max }\right\}$

( $e_{\max }$ present due to persistence)

b. 【Jermaine pictured himself in a tropical paradise】 = $\left\{e_{\text {fantasy }}, e_{\text {pray }}+e_{\text {fantasy }}, \ldots, e_{\text {max }}\right\}$

( $e_{\max }$ present due to persistence) 
c. 【in honor of Janet's wedding $\rrbracket=$
$\left\{e_{\text {pray }}, e_{\text {pray }}+e_{\text {fantasy }}, \ldots, e_{\text {max }}\right\}$
$\left(e_{\max }\right.$ is present due to persistence $)$

d. 【Jermaine prayed in honor of Janet's wedding》 =

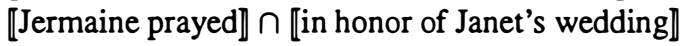

Note in particular that the modifier in (24c) is persistent. The reason for this is that if the denotation in (24a) is persistent, then if we want (24d) to be persistent, the intersective modifier must also be persistent, since intersective a persistent set with a nonpersistent set does not guarantee a persistent result. ${ }^{4}$

Now, if these are our denotations, it's easy to show that some other sentences are incorrectly judged true:

(25) 『Jermaine pictured himself in a tropical paradise

in honor of Janet's wedding》 =

【Jermaine pictured himself in a tropical paradise》 $\cap$

【in honor of Janet's wedding》=

$\left\{e_{\text {pray }}+e_{\text {fantasy }}, e_{\text {max }}, \ldots\right\}$

That is, if we assume (uncontroversially) that truth corresponds to a non-empty event set, then this sentence is (incorrectly) judged true. Note that if we consider $A t(P)$ instead of $P$ for the modifiers and sentence denotations in question, the sentence in (25) would be false, because the elements in the denotation in (25) are a subset of $P-A t(P)$, and without persistence this denotation would be empty.

Similar problems arise in reference in discourse and under quantification. If sentences dynamically add events to context (as in Partee (1984) does in DRT, or Portner (1992) does for a version of Dynamic Montague Grammar), then any element of an existentially quantified event set is eligible for binding or reference. Since $e_{\max }$ is an element of both the denotation in (24a) and the denotation in (24b), both these sentences ought to be equally true:

a. Jermaine prayed. It was in honor of Janet's wedding.

b. Jermaine pictured himself in a tropical paradise. It was in honor of Janet's wedding.

However, they are not. Once again, the presence of elements introduced by persistence compromises intersective behavior. Note again that if the denotations of the sentences in question were $A t(P)$ instead of $P$, no problem would arise, because the elements which induce the incorrect truth values are drawn from $P-A t(P)$.

Both these arguments can be duplicated for quantified NPs, as in (27):

a. In honor of Janet's wedding, every bridesmaid.

b. Every bridesmaid prayed. It was in honor of Janet's wedding. 
Consider a context where all the bridesmaids in Janet's wedding pray, and that in order to honor the wedding, all of them must do it; any less is an affront to the deity in question. In this way, we force the modifier to outscope the quantifier over bridesmaids. Assume further that like Jermaine, these bridesmaids' minds are drifting. It should be easy to see that the above argument can be reconstructed for these cases, and that it is impossible under these assumptions to falsify the examples in (28):

a. In honor of Janet's wedding, every bridesmaid pictured herself in a tropical paradise.

b. Every bridesmaid pictured herself in a tropical paradise. It was in honor of Janet's wedding.

I conclude that in light of these observations, persistence ought to be abandoned as a condition on Davidsonian sentence denotations.

\section{Discussion}

Of course, it's not impossible to preserve persistence in sentence denotations; persistence is merely incompatible with the assumptions I've laid out. ${ }^{5}$ There are alternative accounts of modification and reference which yield a more favorable result. For instance, one might interpret Lasersohn's analogy with information states as requiring persistence only at the sentence level, not below. That is, at every level below $\mathrm{S}$, constituents which denote sets of events denote $\operatorname{At}(P)$ instead of $P$, and the move from $\operatorname{At}(P)$ to $P$ is a matrix-level operation, like existential closure. Under this account, my argument from modification does not arise. One might also argue that my account of event reference is too simplistic, and that the antecedents of event reference may only be drawn from $A t(P)$ for any sentence denotation $P$; this position would counter my second argument.

I can think of at least three replies to this line of counterargument. The first two are methodological. First, in both cases, persistence can be preserved only by eliminating the events which persistence introduces. If persistence is a desirable property of sentence denotations, this strategy strikes me as extremely odd. Second, neither of these responses addresses the fact that persistence still has not been shown to have some demonstrable benefit.

The third argument, I think, is most serious. It seems to me that the two counterarguments put forward are inconsistent with each other. Let's say that the move from $\operatorname{At}(P)$ to $P$ is a matrix-level operation. This means that at some point at the end of the derivation, but before the interpretation is entered into the discourse, the denotation is rendered persistent. However, in order to address the case of elements in $P-A t(P)$ causing problems with reference, we must assume that none of them may be referred to! In other words, the move from $\operatorname{At}(P)$ to $P$ must be postponed in order to circumvent problems with modification, but in order to circumvent problems with reference, we must ignore all the individuals introduced 
by this move anyway. It seems to me that the logical conclusion at this point is not to introduce persistence at all.

One might attempt to reply that Jermaine's praying and daydreaming do not occur at the same spatiotemporal location. This is a possible (although, in my opinion, deeply implausible) reply, but it provides only temporary protection. As long as there are two events which share a spatiotemporal location which cannot share modifiers and preserve truth, one can resurrect the argument I've presented. Consider a case of buying vs. selling. Let's say it's a tradition in Janet's religion for wedding guests to buy a flower from a street vendor on the way to the wedding, and this act of purchasing a flower symbolizes one's support of the worker, one's connection with nature, etc., thereby affirming the couple's place in the world. Jermaine buys a flower with this tradition in mind; however, the street vendor he buys it from hasn't even heard of this religion. It seems to me that (29) is true and (30) is false (remember, it's not the flower itself that's the honor, it's the act of purchasing):

Jermaine bought a flower from a street vendor in honor of Janet's wedding.

\section{A street vendor sold a flower to Jermaine in honor of Janet's wedding.}

I think one would be hard pressed to claim that these events occur at different spatiotemporal locations; if they do not, the argument I've presented above can be reconstructed easily.

One might reply further that perhaps I haven't restricted persistence enough, and that persistence ought to be able to distinguish between these examples. This reply misses the point. One of the properties of persistence which my argument relies on is that it induces "equivalence classes" of events which are determined by the restrictions imposed on persistence. This is because for any set of events which adhere to the restrictions (for instance, for any set of events which share a spatiotemporal location), the lattice-theoretic sum of all the events in the set (that is, $e_{\text {max }}$ ) is always present in the set. So the intersection of any two sets which obey the same set of restrictions will always be nonempty. If these two sets contain events which are conceptually distinct (as in the praying and daydreaming events described above), the problems I outlined above arise. The only way out of this dilemma is to restrict persistence enough so that these ontological transgressions do not arise, but this move would render persistence almost useless from Lasersohn's point of view, since information growth doesn't respect ontological boundaries, but persistence would have to. In other words, if persistence is suitably restricted, it doesn't match the intuitions behind it, and if it isn't suitably restricted, it's wrong.

This result might seem counterintuitive, but I think there's a way to make it perfectly coherent. Information grows through discourse essentially via intersection. That is, we learn more about which of an infinite set of possible worlds we're actually in by intersecting the set of possible worlds in our context with the set of possible worlds denoted by whatever statements we hear (assuming we accept them). The result is a potentially smaller set; that is, we have more information about which world we might be in. Information about individuals in discourse 
grows in much the same way. Let's say someone utters (31), to borrow a standard example from dynamic semantics:

A man walked in. He sat down.

By accepting the first sentence, the hearer accepts the existence of an individual, and knows that individual is a man. By accepting the second sentence, the hearer knows that this individual sat down. In other words, the individual in question is now known to be in the intersection of the set of men and the set of individuals that sat down. In addition, the hearer knows the identity of this individual by virtue not simply of the intersections of the denotations of the descriptions the hearer has been presented, but also by virtue of empty intersections with other denotation sets; so because the hearer knows that the individual in question is a man, and the set of men and the set of women do not overlap, the hearer also knows that the individual in question is not a woman.

Consider how this applies to persistence. One of the main effects of persistence is to make sets of individuals larger; however, a side effect of this growth is that these sets of individuals are now less informative, for two reasons. First, intersecting them with existing sets doesn't have the potential of "shrinking" the result set enough, and second, the "larger" set of individuals might overlap accidently with sets the hearer knows to be nonoverlapping. And this is exactly what we see in the cases above; event sets intersect with other event sets they're not supposed to, and as a result, the framework marks as true statements which aren't. So ironically, a condition which was originally promulgated in service of information preservation actually fatally compromises the orderly growth of information through discourse.

\section{Conclusion}

Clearly, I have not argued that persistence is impossible to maintain. However, I have shown that it's not needed, poorly justified, and in conflict with the most straightforward assumptions one can make about modification and reference. Until some demonstrable benefit can be shown to follow from persistence which cannot be derived otherwise with low cost, it seems to me that persistence ought to be abandoned as a condition on Davidsonian sentence denotations.

\section{Endnotes}

*I would like to thank the SALT 6 attendees for their comments and discussion, especially Chris Barker, Cleo Condoravdi, Tim Fernando, Kai von Fintel, Pauline Jacobson, and Friederike Moltmann. All errors remain mine.

${ }^{1}$ Kratzer assumes that these contextual restrictions are spelled out in the proposition corresponding to the sentence; Lasersohn assumes relevant individuals, but does not formalize the restriction.

${ }^{2}$ For discussion, see Landmian (1993) and Bayer (1995). 
${ }^{3}$ One might point out that the former isn't even possible for the nominalizations all the time, thereby compromising the argument:

(i) *Jermaine/the praying was for ten minutes.

However, this observation fails to recognize that be is only an appropriate copular element in some contexts, and in others it fails (for instance, with path modifiers like into the limousine). When the test makes a distinction, it unambiguously favors the nominalization over the participant.

${ }^{4}$ It is possible that we don't want the intermediate steps to be persistent, which would render this argument invalid; I will address this response in a moment.

${ }^{5}$ Thanks to many discussants who contributed to this clarification, especially von Fintel (p. c.).

\section{References}

Barker, Chris. 1992. Group terms in English: Representing groups as atoms. Journal of Semantics, 9(1):69-93.

Bayer, Samuel. 1995. Confessions of a Lapsed Neo-Davidsonian. Ph.D. thesis, Brown University.

Davidson, Donald. 1967. The logical form of action sentences. In N. Rescher, editor, The Logic of Decision and Action. University of Pittsburgh Press, Pittsburgh.

Kratzer, Angelika. 1989. An investigation of the lumps of thought. Linguistics and Philosophy, 12:607-653.

Krifka, Manfred. 1989. Nominal reference, temporal constitution and quantification in event semantics. In Renate Bartsch et al., editors, Semantics and Contextual Expression. Foris, Dordrecht, pages 75-115.

Krifka, Manfred. 1992. Thematic relations as links between nominal reference and temporal constitution. In Ivan Sag and Anna Szabolcsi, editors, Lexical Matters. University of Chicago Press, Chicago, pages 29-53.

Kripke, Saul. 1965. Semantical analysis of intuitionistic logic. In J. N. Crossley and M. A. E. Dummett, editors, Formal Systems and Recursive Functions. North Holland, Amsterdam.

Landman, Fred. 1986. Towards a Theory of Information. Dordrecht: Foris.

Landman, Fred. 1989. Groups, I. Linguistics and Philosophy, 12(5):559-606.

Landman, Fred. 1993. Events and plurality: The Jerusalem lectures. Manuscript.

Lasersohn, Peter. 1995. Plurality, Conjunction and Events. Dordrecht: Kluwer.

Link, Godehard. 1983. The logical analysis of plurals and mass terms: a latticetheoretical approach. In Rainer Bäuerle et al., editors, Meaning, Use, and Interpretation of Language. Walter de Gruyter, Berlin and New York, pages 302-323.

Ojeda, Almerindo. 1991. Definite descriptions and definite generics. Linguistics and Philosophy, 14(4):367-98. 
Parsons, Terence. 1990. Events in the Semantics of English. Cambridge: MIT Press.

Partee, Barbara. 1984. Nominal and temporal anaphora. Linguistics and Philosophy, 7:243-286.

Portner, Paul. 1992. Situation Theory and the Semantics of Propositional Expressions. Ph.D. thesis, University of Massachusetts, Amherst.

Schein, Barry. 1993. Plurals and Events. Cambridge: MIT Press. 